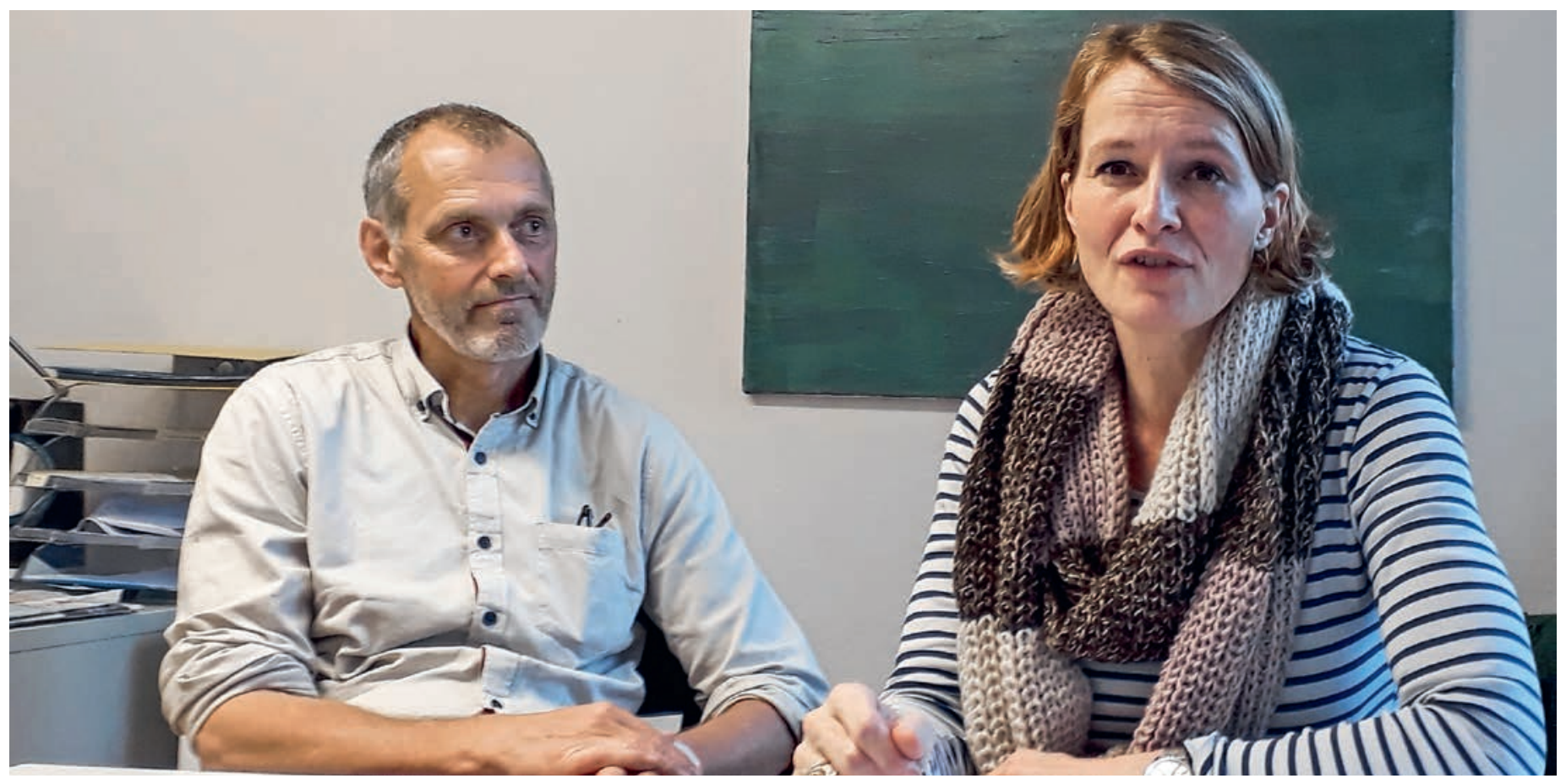

Paul Wittwer (links) und Esther Pauchard.

\title{
Es wird wieder gemordet im Kanton Bern
}

\section{Dominik Heim}

PD Dr. med., Facharzt für Chirurgie, Kompetenzzentrum für Verischerungsmedizin, Suva Luzern, Mitglied FMH

Am Anfang war ein Thema, und plötzlich gab es einen Mord. Der Krimi sei - betonen beide - dann eher Mittel zum Zweck, um das eigentliche Thema dem Leser auf eine «bekömmliche» Art näher zu bringen. Denn schliesslich wolle man ja, dass der Leser an der Geschichte dranbleibe.

Sein erstes Buch, sagt Paul Wittwer, hätte auch ein Roman werden können, aber mit dem Wortspiel «Eiger, Mord (anstelle Mönch) und Jungfrau» (2005) sei dann halt ein Krimi entstanden - ein äusserst erfolgreicher, wie man anmerken muss. So gibt es bei beiden Autoren keine enthaupteten alten Damen oder entstellte Gesichter bei einer Kinderleiche (Silvia Götschi: «Einsiedeln», «Muotothal»), es fehlt auch die fast unerträgliche Brutalität eines Henning Mankell oder eines Jonathan Kellerman. Es sind Geschichten, eingebettet in den normalen Schweizer Alltag, der so normal aber eigentlich gar nicht ist.

\section{«Tödliche Macht»}

Mit Konsternation und Betroffenheit lesen wir, dass sich Menschenhandel und Prostitution - in letzter Zeit vermehrt aus östlichen Ländern - «ganz normal» auch vor unserer Haustüre abspielen. Das lesen wir zwar auch in "20 Minuten», aber dass angesichts der vielen Verflechtungen und gemeinsamen "wirtschaftlichen Interessen» eine Lösung des Problems fast unmöglich erscheint, das realisieren wir dann, wenn wir den neuen Krimi «Tödliche Macht» von Esther Pauchard lesen. Wir erhalten einen Einblick in eine - uns vielleicht eher wenig geläufige - Welt von Zeugenschutz, Identitätswechsel und käuflichem Sex. Wie sagte doch John Lennon: «Woman is the nigger of the world» (1972). Treffender und kürzer kann man es nicht sagen! Und wie ich dann den drei durchaus freundlichen Herren vor kurzem im Zug-Speisewagen zugehört habe, war ich erstaunt festzustellen, wie fest die Geschlechter- 
Zweiklassenwelt noch immer in den Männerköpfen herumschwirrt. Eigentlich sollte man meinen, dass auch der letzte Mann - sofern er ins Kino geht - seit der "Göttlichen Ordnung" (Schweizer Film von Petra Biondina Volpe, 2018) weiss, dass es auch bei uns eine schwer erkämpfte - Gleichberechtigung von Frau und Mann gibt. Ja, man lernt in diesem Pauchard-Buch viel und erschrickt über seine eigene Naivität.

\section{«Bestzeller»}

Das tut man auch im neuen Buch von Paul Wittwer, der vom (naiven?) Traum des Menschen vom Jungbrunnen erzählt. Wie könnte man denn das Altern aufhalten oder mindestens verlangsamen? Ein sehr präsentes Thema heute, und Esther Pauchard bringt es zynisch auf den Punkt und sagt: "Altern und Tod sind heute eigentlich ein Behandlungsfehler.» "Die Antwort ist immer der Tod", zitiert Paul Wittwer den amerikanischen Schriftsteller Philip Roth, der vor kurzem diesem Schicksal auch nicht entrinnen konnte. Und dank Lisa Halliday, die mit dem Schriftsteller in seinen letzten Jahren ein Verhältnis pflegte (bei 45 Jahren Altersunterschied) und seit ihrem Buch «Asymmetry» (2018) ein literarischer VIP ist ("are you game?», fragt er, und sie ist es!), wissen wir auch, wie so ein Altern auch unter der Gürtellinie sein kann. Logisch also, dass man dem Bild von 1957 auf der Schweizer 500-FrankenNote - der Jungbrunnen auf dem Umschlagbild von «Bestzeller» - nachträumt. Das Altern sei eine Krankheit, sagte David Sinclair, Professor für Genetik an der Harvard Medical School, und genau das war für Paul Wittwer der thematische Einstieg in sein Buch «Bestzeller». Nicht unerwartet fragt Johnny, eine seiner Romanfiguren, ob er vielleicht einmal Testosteron versuchen sollte. Dass es wirkt - jedenfalls kurzfristig -, das wissen wir seit Floyd Landis' Tour-de-France-Sieg 2006 und seiner anschliessenden, bitteren Disqualifikation. Ja, konstatiert Paul Wittwer, allzu viel erfahre man in seinem Buch schlussendlich nicht über das Wesen des Alterns, die Demenz, das Pflegeheim, dafür aber umso mehr über die Verflechtung von Medizin und Industrie, wo auch einmal wirtschaftlicher Profit vor echter Wirksam- und Zweckmässigkeit stehen könne. Und ich denke an all die gesponserten Professuren an den Unis, die auch im Buch auf eine bedenkliche Weise dargestellt sind. Hier sei die Medizin auf Abwegen. Die Medizin sei eigentlich kein "rentables Geschäft», sei eben keine Lego-Fabrik! Er kriege das Gefühl nicht los, dass je grösser das Geschäft, desto kleiner die Moral sei. Die medizinische Aufgabe sei es zwar schon, Wünsche zu erfüllen, aber heute würden übertriebene Hoffnungen in die Medizin gesetzt. Verzicht sei zum Fremdwort ge- worden. Ja, pflichtet ihm Esther Pauchard bei, man wolle halt einfach den 5er und das Weggli, möglichst alles für möglichst wenig. Die «Aldiformel», kann ich noch einwerfen.

\section{Die eigene Themenwahl}

Und so wird bei beiden klar, dass sie ihr Thema selbst aussuchen, und dass kein Grossverlag dahinter steckt, der den vorgeschobenen Bonus zu abgemachten Terminen wieder hereinwirtschaften möchte. Man lese dazu "Camino Island» des US-amerikanischen Bestsellerautors John Grisham: Die Geschichte der jungen Schriftstellerin, die nach dem Erfolg ihres Erstlings eine Schreibblockade hat und finanziell wohl in den Ruin liefe, wäre da nicht der raffinierte Raub von Scott Fitzgeralds Originalmanuskripten (darunter natürlich auch «The Great Gatsby») ...

Es sei schön, betonen beide, nicht vom Schreiben leben zu müssen. Esther Pauchard findet ihre Themen im psychiatrischen Umfeld (Drogenprostitution) und ist so mit dem Thema käuflicher Sex in «Tödliche Macht» sehr vertraut. Paul Wittwer findet seine Geschichten im Hausarzt-Alltag und beschreibt den Schreibvorgang als: «Man beschäftigt sich damit, denkt nach und sitzt wieder hin, und das Bedürfnis, selbst etwas zu schreiben, überkommt einen plötzlich.» Esther Pauchard pflichtet dem bei: «Es fägt, und irgendwann schreibt es sich dann ganz von selbst.» Plötzlich gebe es Platz für Kreativität im Alltag. Mut brauche es aber schon zum Schreiben. Darum habe sie den Anfang sehr gerne, weil dann das bis anhin geschriebene Buch allein ihr gehöre. Ja, meint Paul Wittwer, der dann folgende Lektoratsprozess sei schmerzhaft. Und wenn das Buch dann draussen ist, gehört es eben nicht mehr mir, sondern allen, schiebt Esther Pauchard nach. Übrigens, studierte Literaten/Schriftsteller seien sie beileibe keine, ja, sie hätten es eigentlich gar nicht so gerne, wenn man sie als Schriftsteller/Autoren bezeichne.

\section{Die Zeit nach dem Buch}

Mit dem Schreiben ist es aber nicht getan. Esther Pauchard erklärte dies an ihrer Buchvernissage im September: Sie spüre dann jeweils so ein metaphysisches Grümseln beim Manuskript-Druck - jetzt ist es da, das Buch! Und so verändere dieser Moment den Autor und auch die Zeit nach der Publikation: Im zweiten Jahr schreibe sie wieder, das erste aber gehöre den Lesungen und andern PR-Anlässen. Sie habe sich jetzt auf ungefähr eine Lesung pro Woche eingestellt. Nein, das sei ihm dann doch zu viel, sagt Paul Wittwer, er sage dann halt einfach ab. Schliesslich sei mit dem Schreiben 
keine Exit-Türe aus der Medizin gesucht; Beruf und Familie hätten da immer noch Vorrang. Und man merkt, er braucht deutlich länger für ein Buch als Esther Pauchard. Sie ist schon fast in einem Zweijahresrhythmus, bemüht sich aber trotzdem, sich dabei auch Freiräume zu schaffen. So kommt sie im Jahr auf rund 30 Lesungen, wobei sie grosse Lesungen gar nicht so gerne habe, viel lieber diese kleinen, fast familiären Anlässe. Auch Kolumnen schreibe sie gerne, es müsse nicht grad immer ein neues Buch sein. Die Kombination von Psychiatrie und Schreiben, die Kombination von Arztberuf und Autor, das sei einfach spannend. Auch bei Paul Wittwer muss es nicht grad immer ein neues Buch sein, sehr genossen habe er es, als er einmal am Hausarztkongress in Arosa über die narrative Medizin berichten konnte. Und so ergebe das Schreiben auch Kontakte, die nicht direkt buchbezogen seien, man trage auch Bücher an ihn heran, wolle seine Meinung wissen. Wichtig ist beiden, dass sie mit ihrer Schreibarbeit Denkanstösse geben können, und das tun sie mit ihren Themen in der Tat!

\section{Die unweigerliche Frage zum Schluss}

So gegen Ende eines Interviews kommt dann (unweigerlich) die Frage nach dem, was nun kommen wird. Das ist auch der Moment, wo der Interviewpartner sieht, dass sein Gegenüber unruhig seine Notizen checkt, sich vergewissert, dass er auch ja nichts vergessen hat. Vom Filmregisseur will man wissen, welchen Film er als Nächstes drehen will/wird. Vom Musiker, ob er bei der Stilrichtung bleibt, und beim Schriftsteller, welches Thema er als Nächstes behandeln möchte. In diesem Gespräch kam dieses Thema allerdings schon viel früher, nämlich als sich die beiden zur gegenwärtigen Situation der Medizin in der heutigen Gesellschaft kritisch äusserten. Am Leuchten in den Augen von Esther Pauchard merkte man genau, dass sie nicht nur darüber diskutieren möchte, sondern, dass sie da schon einen Plan hat. Sie, die immer akribisch schon vor dem Schreiben recherchiert vom Hotel im Engadin bis zum Helvetiaplatz in Zürich (das Recherchieren benötige ruhig einmal ein halbes Jahr und könne schon ein halbes Buch füllen), weiss da schon gewisse Namen, die sie "vor dem Mikrofon" haben möchte. Verwundert es, dass zu einer Zeit, da die grösste Sorge der Bevölkerung der eigenen Gesundheit (und auch dem unweigerlichen Altern) gilt, die jetzige Gesundheitspolitik ihr neues, nicht direkt medizinisches Arbeitsfeld sein wird? Man kann sich schon gut vorstellen, wie sie ihr Gegenüber mit ihren Fragen löchert, denn schliesslich lerne man dabei auch immer, so quasi eine eigene Horizonterweiterung. Für Paul Wittwer darf es auch einmal etwas ganz anderes als ein Krimi sein. Er schmunzelt, wenn er sagt, dass er sich durchaus auch vorstellen könnte, einmal ein Buch in Mundart zu schreiben, so im schönen Emmentalerdialekt. Und man merkt, dass das Schreiben eben keine Pflicht, sondern eigentlich ein Moment des Geniessens ist. Kein musikalischer Stilwechsel also, aber ein grammatikalischer? Ein Blick auf die Uhr, nein, wir haben keine Gesprächsdauer abgemacht, aber vielleicht muss Esther Pauchard noch ihre medizinische Arbeit in der Klinik Selhofen in Burgdorf erledigen. Man kann ja nicht nur schreiben! Ich verabschiede mich vom schelmisch lachenden Paul Wittwer und der fröhlichen, impulsiven Esther Pauchard und frage mich, ob es bei Paul Wittwer als Nächstes wirklich einen Dialektroman gibt und ob Kassandra, die abgeklärte, psychiatrische Protagonistin in den ersten drei Büchern von Esther Pauchard, und Melissa Braun, die immer aufbrausende, medizinische Praxisassistentin, als Protagonistin in den zwei letzten Büchern, den «Rank zusammen finden». Mit dieser, meiner, Interpretation des Charakters von Melissa ist die Autorin übrigens gar nicht einverstanden Paul Wittwer schmunzelt wieder -, aber die Freiheit des Lesers ist halt grenzenlos. Ja, das dürfe - sagt sie schon verraten werden: Kassandra kommt zurück! Und nein, sie, Esther Pauchard, sei nicht Kassandra!

Bildnachweis

Foto: Dominik Heim
Ihre Neuerscheinungen

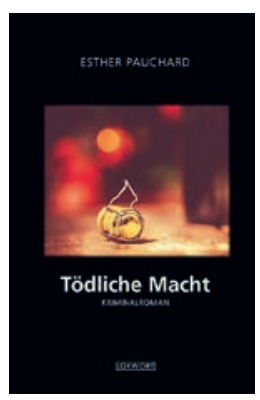

Esther Pauchard

Tödliche Macht

Kriminalroman

336 Seiten

Klappenbroschur

Bern: Lokwort Buchverlag; 2018

ISBN 978-3-906806-19-8

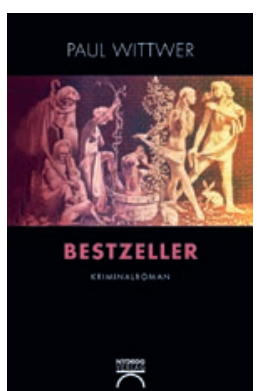

Paul Wittwer

Bestzeller

Kriminalroman

432 Seiten

Gebundene Ausgabe:

Bern: Nydegg Verlag; 2018

ISBN 978-3905961164 